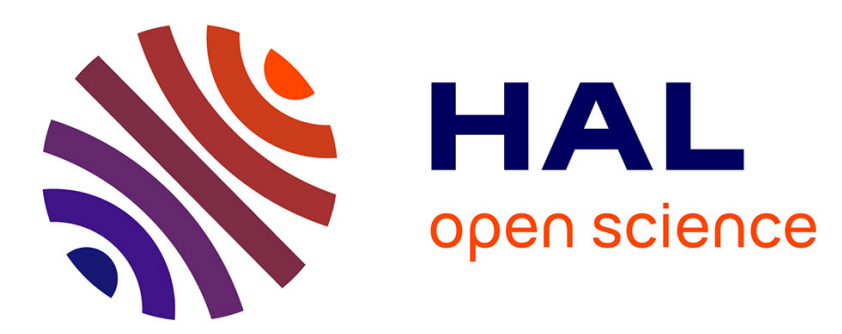

\title{
La direction des sociétés anonymes depuis la fin du XIXe siècle: le droit entretient la confusion des pratiques
}

Hervé Joly

\section{To cite this version:}

Hervé Joly. La direction des sociétés anonymes depuis la fin du XIXe siècle: le droit entretient la confusion des pratiques. Entreprises et Histoire, 2009, 57, pp.111-125. halshs-00536675

\section{HAL Id: halshs-00536675 \\ https://shs.hal.science/halshs-00536675}

Submitted on 4 Aug 2011

HAL is a multi-disciplinary open access archive for the deposit and dissemination of scientific research documents, whether they are published or not. The documents may come from teaching and research institutions in France or abroad, or from public or private research centers.
L'archive ouverte pluridisciplinaire HAL, est destinée au dépôt et à la diffusion de documents scientifiques de niveau recherche, publiés ou non, émanant des établissements d'enseignement et de recherche français ou étrangers, des laboratoires publics ou privés. 


\title{
LA DIRECTION DES SOCIÉTÉS ANONYMES DEPUIS LA FIN DU XIX ${ }^{\mathrm{E}}$ SIËCLE : LE DROIT ENTRETIENT LA CONFUSION DES PRATIQUES
}

\author{
par Hervé JOLY \\ LARHRA \\ CNRS-Université de Lyon
}

Qu'est-ce qu'un dirigeant en droit et qui peut prétendre à le nommer ou à le contrôler ? L'examen de la naissance de la fonction dirigeante en France montre que celle-ci est née du croisement de deux fonctions : I'administration de la société d'une part et la direction des opérations sur le terrain d'autre part. Au lieu de distinguer les deux fonctions de gestion et de régler leurs rapports, le droit les a confiées toutes les deux au conseil d'administration, puis il a même créé, sous Vichy, la fonction double de PDG. L'article revient sur les raisons de cette fusion, et en souligne la confusion.

Dans une entreprise personnelle, le patron est le propriétaire, quelle que soit ensuite la manière dont il gère son affaire et confie des responsabilités à ses collaborateurs salariés. Dans une société, les choses se compliquent parce qu'il y a par définition plusieurs associés - sous réserve de la forme introduite récemment en droit français (1985) de la société unipersonnelle à responsabilité limitée. Dans la forme la plus simple, la société en nom collectif (SNC), ils sont en nombre limité, deux, trois ou quatre en pratique, et sont supposés se partager la direction de l'entreprise. Il arrive cependant que les statuts prévoient explicitement qu'un associé puisse, à la différence des autres, ne pas consacrer tout son temps à la gestion de l'affaire ; c'est le cas, par exemple, lorsque l'apporteur d'une entreprise personnelle pré- pare sa succession en associant ses enfants ou ses collaborateurs. La transformation ultérieure en société en commandite simple (SCS) par l'adjonction de commanditaires, simples détenteurs de parts sans droit de regard sur la gestion, ne change rien à la gestion partagée entre les associés commandités. Dans la seule forme de société par actions autorisée librement par le Code de commerce de 1807, la société en commandite par actions (SCA), les statuts désignent formellement des associés gérants qui disposent des pouvoirs de gestion; les autres actionnaires anonymes pouvant simplement être représentés dans un conseil de surveillance. Il en est de même dans la nouvelle société à responsabilité limitée (SARL) introduite en droit français par la loi de 1925, avec des gérants statutaires désignés parmi 
les porteurs de parts. En revanche, la société anonyme (SA), introduite sous réserve d'autorisation préalable en 1807 et libéralisée par la loi de 1867 , reste effectivement en principe anonyme dans ses statuts quant à la désignation des dirigeants. Des fondateurs existent bien nominalement en tant que rédacteurs des textes constitutifs et organisateurs de l'appel à souscription au capital originel, mais ils ne peuvent se garantir durablement une position dirigeante dans la société qui doit pouvoir exister sans eux ${ }^{1}$. La SA comprend, d'une part, une collectivité - plus ou moins large selon le degré d'ouverture au public de la souscription, plus ou moins équilibrée selon l'importance des apports effectués par les uns et par les autres - d' " associés », détenteurs à un moment donné d'actions, une qualité susceptible de se perdre ou de se gagner par une simple transaction non déclarée. D'autre part, l'entreprise exploitée en SA emploie un certain nombre de collaborateurs salariés, liés à elle par un « contrat de louage de services» ou contrat de travail. L'identification des dirigeants des SA se joue donc au croisement de ces deux collectivités. La formulation succincte du Code de commerce de 1807 montre bien cette ambiguitté : la SA « est administrée par des mandataires à temps, révocables, associés ou non-associés, salariés ou gratuits ». C'est la pratique ultérieure, sur laquelle l'État disposait, avec l'autorisation requise du Conseil d'État pour la création, d'un droit de regard important, qui a précisé les modalités ${ }^{2}$. La loi de 1867 reprend en gros ces dispositions dans un article unique qui accentue encore la confusion. Différentes configurations restent pos- sibles, d'autant que le filtre de l'autorisation préalable du Conseil d'État disparaît. Une grande liberté contractuelle est laissée aux associés ; la manière dont ils s'organisent entre eux n'est pour l'essentiel pas considérée comme d'ordre public. Il a fallu le contexte politique particulier du régime de Vichy pour imposer une réglementation contraignante. Mais, comme le montre l'étude des pratiques adoptées par les grandes entreprises ${ }^{3}$, cette nouvelle législation, plutôt qu'une rationalisation des pratiques, a accentué la confusion ; les configurations sont restées plurielles et les évolutions législatives ultérieures les ont encore multipliées.

\section{UNE SIMPLE ESQUISSE DES FONCTIONS DE DIRECTION PROPOSÉE PAR LA LOI DE 1867}

Les règles fixées par la loi de 1867, qui complètent à peine celles de 1807, laissent subsister un grand flou des pratiques : elles se contentent de prévoir que «les sociétés anonymes sont administrées par un ou plusieurs mandataires à temps, révocables, salariés ou gratuits, pris parmi les associés », tout en prévoyant, pour la première fois, explicitement la désignation d'un « directeur» (art. 22).

\section{L'existence de fait d'un conseil d'administration collégial}

Jusqu'en 1940, l'existence d'un « conseil d'administration » rassemblant les « manda-

\footnotetext{
'La loi de 1867 les autorisait certes à s'attribuer, pratique fréquente, des avantages particuliers par le mécanisme des parts de fondateur, mais ceux-ci ne concernent que la répartition des profits et pas l'administration de la société.

${ }^{2}$ Sur l'importance, relative selon les époques, de l'harmonisation imposée par le Conseil d'État, voir C. E. Freedeman, Joint-stock enterprise in France, 1807-1867: from privileged company to modern corporation, Chapel Hill, University of North Carolina Press, 1979 et A. Lefebvre-Teillard, La société anonyme au XIXe siècle : du Code de commerce à la loi de 1867, histoire d'un instrument juridique du développement capitaliste, Paris, PUF, 1985.

${ }^{3}$ L'article s'appuie, pour l'essentiel, sur un corpus de 21 grands groupes industriels dont l'organisation et la composition des organes dirigeants des sociétés mères ont été étudiées systématiquement jusqu'en 1966 : voir H. Joly, Diriger une grande entreprise française au XX $X^{e}$ siècle : modes de gouvernance, trajectoires et recrutement, mémoire inédit d'habilitation à diriger les recherches en histoire, EHESS, 2008, consultable en ligne sur le site TEL http://tel.archives-ouvertes.fr/tel-00343525/en/.
} 
taires » désignés par les actionnaires pour administrer la SA est restée ignorée par la législation. Elle n'est d'ailleurs pas obligatoire : la loi de 1867 n'exclut pas la solution d'un « mandataire » unique. Toutes les sociétés anonymes importantes ont cependant une pluralité d'administrateurs, avec un effectif minimum fixé par les statuts souvent de sept voire neuf (Compagnie française des métaux en 1892, Thomson-Houston en 1893, etc.) membres dès l'origine. La seule exception connue pour une grande entreprise est celle des Fonderies de Pont-à-Mousson : lors de la transformation de l'ancienne SCA en SA en 1886, le directeur Xavier Rogé, entre-temps devenu l'actionnaire dominant ${ }^{4}$, est le seul administrateur désigné. En 1899, son adjoint Camille Cavallier le rejoint, mais, après le décès du premier un an plus tard, le second devient à son tour administrateur unique jusqu'en $1917^{5}$. Il ne s'agit pas encore d'une très grande entreprise ; les directeurs successifs parviennent, sans fortune personnelle préalable, à partir de leurs seuls émoluments, à acquérir une position dominante au sein d'un capital restreint. Partout ailleurs, la pratique d'un conseil collégial s'est installée. Même lorsque le capital est majoritairement concentré au sein d'une famille par exemple, elle permet d'associer à l'administration les différents héritiers ou des partenaires extérieurs, sans que ceux-ci soient nécessairement des actionnaires importants.

\section{L'exigence formelle de la qualité d'actionnaire}

$$
\text { L'exigence nouvelle de la loi de } 1867 \text { que }
$$
les «mandataires » administrant la société soient actionnaires s'avère une contrainte très limitée. Le législateur a renoncé à fixer un seuil minimal de détention tel qu'il avait existé dans la loi de 1863 qui avait introduit une première société à responsabilité limitée ${ }^{6}$. Le nombre d'actions dites «de garantie », parce qu'elles sont supposées garantir la gestion des administrateurs en cas de faute, à détenir est fixé librement par les statuts et peut ne pas dépasser une unité. En pratique, le nombre exigé est souvent plus élevé mais il ne représente pas un véritable obstacle pour l'accès au conseil ; les barrières sociales et professionnelles de fait sont beaucoup plus fortes. La doctrine juridique admet de toute façon que la détention de ces actions ne soit pas une condition préalable à l'élection comme administrateur ${ }^{7}$. L'administrateur peut ne régulariser sa situation qu'après coup. Si chez Saint-Gobain le seuil est élevé, cela reflète plus largement le fait que le cercle des actionnaires est longtemps luimême étroit : le capital n'est divisé qu'entre 4600 actions d'une valeur moyenne de $32000 \mathrm{~F}$ chacune jusqu'en $1907^{8}$. Il n'y a à cette date que 1295 actionnaires et, parmi eux, seule une minorité détient les cinq actions nécessaires pour voter à l'assemblée générale et, a fortiori, les dix requises pour siéger au conseil. Pouvoir immobiliser $320000 \mathrm{~F}$ dans une seule affaire réserve bien sûr cette fonction à des membres de la bourgeoisie ou de l'aristocratie fortunée, mais il reste plus de prétendants possibles que d'élus. Le soyeux lyonnais Auguste Isaac s'inquiète bien dans son journal de devoir, après son élection en 1910 au conseil de la Compagnie universelle du canal maritime de Suez, consacrer $520000 \mathrm{~F}$ à l'achat de 100

\footnotetext{
${ }^{4}$ J.-M. Moine, Les barons du fer. Les maîtres de forges en Lorraine du milieu du 19 e siècle aux années trente. Histoire sociale d'un patronat métallurgique, Metz-Nancy, Serpenoise-Presses Universitaires de Nancy, 1989, p. 83.

${ }_{5}^{5}$ A. Baudant, Pont-à-Mousson (1918-1939). Stratégies industrielles d'une dynastie lorraine, Paris, Publications de la Sorbonne, 1980, p. 3-7.

${ }^{6}$ P. Villard, Des administrateurs dans les sociétés anonymes, Paris, F. Pichon, 1884, p. 12.

${ }^{7} \mathrm{C}$. Houpin et H. Bosvieux, Traité général théorique et pratique des sociétés civiles et commerciales et des associations, Paris, Sirey, 1935, t. I, p. 205.

${ }^{8}$ J.-P. Daviet, Un destin international. La Compagnie de Saint-Gobain de 1830 à 1939, Paris, Éditions des Archives contemporaines, 1988, p. 222.
} 
actions de garantie, mais il s'y résout vite après avoir calculé que cela lui rapportera, entre jetons de présence et dividende, 45000 $\mathrm{F}$ par an, soit un placement d'un rendement inégalable à $8,65 \% \%^{9}$.. Cette exigence légale purement formelle pour un administrateur d'être actionnaire a été maintenue jusqu'à la loi récente «de modernisation de l'économie » du 4 août 2008 qui laisse dorénavant la liberté aux statuts d'en prévoir ou non l'obligation (art. L. 2225-25, al. 2 du Code de commerce).

\section{Administrateurs et salariés}

La loi de 1867 reprend la possibilité explicite du Code de commerce de 1807 que l'administrateur soit par ailleurs salarié de l'entreprise. Il ne s'agit bien sûr pas dans l'esprit du législateur de l'époque d'assurer une représentation au conseil de l'ensemble du personnel salarié telle qu'on l'entend aujourd'hui ; cette opportunité concerne plutôt, pour employer un terme anachronique, des «cadres » de l'entreprise, directeurs ou fondés de pouvoir en particulier. Elle introduit une première confusion entre administration et gestion de l'entreprise ; les administrateurs ne sont pas de simples surveillants extérieurs à la gestion quotidienne ; ils peuvent y être directement associés et avoir un lien de subordination avec l'entreprise qui les emploie, ce qui pose la question toujours très actuelle de leur indépendance. Rien n'est prévu quant au lien entre ces deux statuts : dans la mesure où la qualité de salarié n'est qu'une faculté, la perte de l'un n'est pas supposée entraîner la perte de l'autre, même s'il paraît difficile qu'un salarié licencié reste administrateur de la société qui l'employait. L'alternative du texte entre «salariés ou gratuits » ne correspond pas non plus à la pratique : les administrateurs, même s'ils ne sont pas salariés de la société, n'exercent pas leur fonction gratuitement : ils reçoivent des rémunérations spécifiques qui peuvent être importantes, sous la forme à la fois de tantièmes (part des bénéfices prévue par les statuts) et de jetons de présence.

\section{L'exigence de révocabilité}

La seule exigence légale impérative est celle de la révocabilité des administrateurs. Sa portée est cependant discutée par la doctrine. Est clairement exclue toute disposition des statuts qui, à l'image des gérants d'une commandite, installerait les administrateurs durablement dans leur position. Même ceux éventuellement nommés par les statuts d'origine peuvent être écartés par un simple vote de l'assemblée générale ordinaire ${ }^{10}$. De manière générale, aucune condition supplémentaire à celle d'une majorité simple des suffrages exprimés des actionnaires présents ne peut être exigée ; cela reviendrait à rapprocher la révocation des conditions d'une assemblée générale extraordinaire, et donc à donner une position statutaire aux administrateurs. La loi de 1867 prévoit simplement que les premiers administrateurs sont élus pour un maximum de six ans, et de trois ans seulement pour ceux désignés sans élection par les statuts d'origine (art. 25, al. 2). En pratique, cette durée de six ans est généralement adoptée, avec un renouvellement par tiers tous les deux ans pour éviter d'avoir à élire ou réélire tous les administrateurs en même temps. Mais les actionnaires gardent toujours la possibilité d'interrompre ce mandat avant son terme. Dans l'autre sens, la loi n'interdit pas une clause de non-rééligibilité pour les administrateurs sortants («sont rééligibles, sauf disposition contraire ») ${ }^{11}$, mais cette possibilité n'est presque jamais utilisée en pratique : il paraît difficile d'écar-

\footnotetext{
${ }^{9}$ Voir, au 22 avril 1910, A. Isaac, Journal d'un notable lyonnais 1906-1933 (textes choisis et annotés par H. Joly), Lyon, BGA Permezel, 2002, p. 126-127.

${ }^{10}$ P. Villard, Des administrateurs..., op. cit., p. 27.

${ }^{11}$ Ibid., p. 24.
} 
ter du conseil un gros actionnaire individuel. Plus discutée par les juristes est la question de savoir si une éventuelle révocation peut s'accompagner d'indemnités. Selon une position stricte, tout obstacle financier qui pourrait contribuer à dissuader les actionnaires d'exercer leur droit à révocabilité doit être écartét ${ }^{2}$. D'autres auteurs considèrent cependant que « si la révocation était inspirée par l'intention de nuire à ces mandataires ou si elle était accompagnée de mesures de nature à porter injustement atteinte à leur honneur et à les discréditer, il pourrait y avoir dans la manière même dont le droit de révocation serait exercé la source d'un délit ou d'un quasi-délit donnant naissance à une action en dommages-intérêts $\gg^{13}$. La question est compliquée par l'existence parallèle éventuelle d'un contrat de travail dont la rupture logiquement concomitante peut déboucher sur des indemnités. À l'inverse, la loi n'interdit pas aux administrateurs de démissionner à leur guise, sans qu'ils aient à motiver ou à justifier leur renonciation, sous réserve que « la démission ne doit pas être intempestive ou inopportune, sinon la société pourrait se faire indemniser du préjudice $»^{14}$.

La révocation pure et simple d'un administrateur par l'assemblée générale est de toute façon exceptionnelle : le cas en 1899 de cinq administrateurs de la Compagnie française des métaux accusés d'avoir approuvé une vente à découvert de stocks de cuivre qui a occasionné de lourdes pertes pour la société apparaît à peu près unique en son genre ${ }^{15}$. On peine à trouver d'autres exemples historiques d'une crise aussi ouverte dans de grandes entreprises : même les administrateurs du groupe bancaire Oustric, qui ont entraîné les Automobiles Peugeot dans une très grave crise financière, sont simplement amenés à démissionner du conseil en $1930^{16}$. Il en est de même pour André Vincent aux Aciéries de Firminy un an plus tard, à la suite de la grave défaillance de l'entreprise qu'il dirigeait, le Comptoir Lyon-Alemand, qui jouait le rôle de banquier du groupe ${ }^{17}$.

En l'absence jusqu'aux années 1960 de limite d'âge aussi bien légale que statutaire, la qualité d'administrateur ne se perd souvent que par le décès ; même des administrateurs durablement empêchés de siéger par la maladie répugnent à abandonner leurs mandats et les revenus associés. De manière générale, lorsqu'il y a un conflit personnel, l'administrateur remet sa démission de manière plus ou moins contrainte. À la Société chimique des usines du Rhône, l'un des cofondateurs, Prosper Monnet, déjà écarté de la direction des usines en octobre 1899, est sommé en février suivant de s'expliquer sur les intérêts qu'il aurait pris dans une nouvelle entreprise chimique lyonnaise concurrente ${ }^{18}$. En août, il se voit accusé d'avoir colporté « des bruits défavorables sur la situation intérieure de la société sur la place » de Lyon. En mars 1901, il refuse la réduction de ses émoluments que le conseil lui a imposée en son absence en décembre. Finalement, l'affaire se termine en juin par une simple non-proposition de réélection à l'assemblée générale, son mandat venant opportunément à échéance. De tels conflits au sein du conseil interviennent surtout dans les premières années de fonc-

\footnotetext{
${ }^{12}$ Ibid., p. 28.

${ }^{13}$ P. Gontier, Le directeur et l'administrateur délégué de la société anonyme, Paris, Giard \& Brière, 2 éd., 1915, p. 20 .

${ }^{14}$ P. Villard, Des administrateurs..., op. cit., p. 26.

${ }^{15}$ Archives nationales du monde du travail, Roubaix, 65 AQ K56 ${ }^{1}$, Compagnie française des métaux, rapport annuel (RA), assemblée générale (AG) 30 juin 1899.

${ }^{16} \mathrm{Au} 1^{\mathrm{er}}$ novembre 1930. Archives historiques groupe Crédit agricole, fonds du Crédit lyonnais (ACL), DEEF 41086, RA Automobiles Peugeot, exercice 1929-1930, AG 4 avril 1931.

${ }^{17}$ Démissionnaire au 6 octobre 1931. ACL, DEEF 41304, RA Aciéries de Firminy, exercice 1930-1931.

${ }^{18}$ Archives départementales du Rhône (ADR), 146 J7, fonds Cayez, Procès-verbal (PV) du conseil d'administration (CA), Société chimique des usines du Rhône, séances du 21 octobre 1899 au 24 juin 1901.
} 
tionnement de la société, à un moment où les responsabilités des uns ou des autres sont encore mal établies. L'hypothèse ultérieure la plus fréquente à l'origine d'un départ prématuré est celle d'une évolution de la composition du capital qui remet en cause la présence d'un administrateur ; si celui-ci est souvent amené à se retirer du conseil après la cession des actions qu'il représentait, son départ ne relève pas d'une révocation formelle.

\section{L'exception nécessaire à la collégialité}

Le Code de commerce de 1807 ne comportait aucune exception à la collégialité des administrateurs. À la différence des associés d'une société de personnes ou des gérants d'une commandite, les statuts ne prévoient cependant pas de clause d'exclusivité selon laquelle ils devraient consacrer l'essentiel de leur temps à l'administration de la société. Jusqu'en 1940, il n'existe aucune restriction légale au cumul de mandats d'administrateurs et certaines personnalités en ont accumulé plusieurs dizaines, à l'image d'un Gabriel Cordier (1865-1934), pour lequel un relevé non nécessairement exhaustif dans l'édition 1928 de l'Annuaire Chaix des sociétés par actions permet d'en recenser 19. Ces cumuls sont très critiqués par l'opinion publique dans le contexte de la crise des années 1930 parce qu'ils sont suspectés d'être motivés par le seul appât du gain ; un contrôle effectif de la gestion des différentes entreprises concernées paraît impossible. Cumulard ou pas, l'administrateur n'est de toute façon pas nécessairement en mesure de consacrer tout son temps à la société. Si des incompatibilités sont progressivement intro- duites avec certaines professions libérales (notaires, avocats au barreau de Paris) ou publiques (militaires, agents du ministère des Affaires étrangères), il peut être parlementaire, fonctionnaire d'autres ministères ou surtout cadre salarié d'une autre entreprise ${ }^{19}$. L'administration de la société ne peut être pour lui qu'une activité annexe, a fortiori lorsqu'elle se partage entre plusieurs sociétés. En pratique, l'activité d'administrateur s'exerce principalement à l'occasion de réunions du conseil qui se tiennent à un rythme très variable, mais qui n'est jamais quoti$\operatorname{dien}^{20}$. Les statuts se contentent de prévoir que «le conseil se réunit aussi souvent que l'intérêt de la société l'exige », avec la mention éventuelle d'une fréquence minimale généralement mensuelle. Si ce rythme est souvent plus ou moins adopté, on trouve aussi des fréquences plus élevées. Le conseil d'administration de la Société électro-métallurgique française de Froges (Isère) se réunit à 9 reprises au cours de ses quinze premiers jours de fonctionnement du 26 octobre au 11 novembre 1888, 28 fois au cours de la première année d'exercice et 82 fois pendant les quatre premières, soit en moyenne de manière quasi bimensuelle ${ }^{21}$. Saint-Gobain a encore, malgré son ancienneté, un rythme exceptionnellement élevé jusqu'aux années 1930 avec, à partir de 1901, deux séances hebdomadaires consacrées à chacune des branches (glaces et produits chimiques, les « affaires générales » étant traitées également dans l'une des deux séances) $)^{22}$. À partir de 1933, les deux séances de branches n'ont plus lieu qu'une semaine sur deux, avant que la pratique d'une séance unique tous les quinze jours ne soit adoptée en 1935. À une époque où les déplacements ne se font pas à la vitesse de l'avion ou du TGV, une telle fréquence

\footnotetext{
${ }^{19}$ C. Houpin et H. Bosvieux, Traité général..., op. cit., p. 209.

${ }^{20} \mathrm{Cf}$. P. Maureil-Deschamps, De la situation respective du conseil d'administration et du directeur dans la société anonyme, thèse de doctorat, faculté de droit de Paris, Paris, Imprimerie des Facultés A. Michalon, 1909, p. 17, qui évoque même des fréquences trimestrielles, voire annuelles dans certaines sociétés.

${ }^{21}$ Archives Pechiney, 072-13-29967, PV du CA de la SEMF.

${ }^{22}$ Archives Saint-Gobain (ASG), série 2B 26 (1928-1931 et suivantes), PV du CA de Saint-Gobain.
} 
n'est possible que tant que les administrateurs, issus des mêmes milieux financiers, ont eu une origine géographique commune. L'installation du siège social à Paris est certes susceptible de favoriser la fréquentation des réunions, mais il se trouve souvent des administrateurs provinciaux, voire étrangers suisses ou, avant 1914, allemands en particulier - qui ne peuvent se déplacer si souvent. À Saint-Gobain, c'est aussi le profil des administrateurs qui fait exception : ils appartiennent souvent à des familles de rentiers dont l'engagement dans l'entreprise est assez exclusif ; ils ne cumulent guère d'autres mandats. Mais quel que soit le rythme des réunions, même élevé, il ne s'agit toujours que d'une présence ponctuelle et pas d'un suivi quotidien de la gestion. En pratique, l'administrateur n'a souvent pas de bureau et de secrétariat propres au siège de la société. Il est donc extérieur à l'entreprise au sens physique du terme. Ses revenus, même s'ils peuvent être élevés, n'assurent pas une rémunération fixe. Les jetons de présence ne sont que des indemnités relativement modestes et les tantièmes restent suspendus au caractère bénéficiaire de l'affaire.

\section{Des administrateurs plus investis que les autres}

Dans le silence de la loi jusqu'en 1867, et dans le flou ensuite jusqu'en 1940, sont apparus des administrateurs particuliers, plus investis que les autres dans la gestion de l'entreprise. L'existence d'un conseil d'administration a d'abord débouché logiquement sur la désignation, généralement prévue par les statuts, d'un président, souvent flanqué d'un ou plusieurs vice-présidents pour le suppléer. Sa prérogative minimale est au moins de convoquer ses collègues aux séances, d'en fixer l'ordre du jour et de les présider; il est aussi celui qui préside les assemblées générales. Son rôle peut aussi dans les faits s'étendre à celui d'un porte-parole de l'entreprise à l'extérieur. Si cette fonction entraîne un rapprochement de son titulaire avec l'en- treprise, concrétisé par l'obtention d'un bureau et d'un secrétariat personnels, l'engagement n'en devient pas pour autant exclusif. On trouve d'ailleurs des cas spectaculaires de cumuls : Gabriel Cordier est ainsi, jusqu'à sa mort en 1934, président de l'Énergie électrique du littoral méditerranéen (EELM), d'Alais, Froges \& Camargue (AFC) et de la Compagnie des chemins de fer du PLM ; Ernest Mercier préside la Lyonnaise des eaux et de l'éclairage, l'Union d'électricité, Alsthom et la Compagnie française des pétroles dans les années 1930, etc. Là encore, la seule fonction de président ne donne pas nécessairement droit, il est vrai, à un salaire fixe ; le titulaire reçoit simplement une part plus importante, double par exemple, des jetons de présence et des tantièmes.

Une autre manière pour certains administrateurs d'être plus investis que d'autres est l'existence, organisée dans le silence de la loi et même souvent des statuts par le conseil d'administration en son sein, d'un organe plus restreint, généralement appelé «comité de direction », qui rassemble habituellement le président, le ou les vice-présidents et un ou deux autres administrateurs plus disponibles. Cet organe ne dispose pas formellement de prérogatives spécifiques, mais il se réunit plus souvent que le conseil, par exemple de manière hebdomadaire, ce qui lui permet un suivi plus régulier de la gestion; il prépare les décisions du conseil qui les adopte ensuite. Là encore, ces fonctions s'accompagnent généralement de rémunérations supérieures, mais elles restent non exclusives.

\section{L'émergence du directeur envisagée par la loi de 1867}

Il est vite apparu que l'administration d'une entreprise importante exploitée en SA impliquait la présence régulière d'au moins un «directeur», en mesure de donner des directives et de suivre au quotidien l'ensemble des services et établissements. Se pose alors la question de savoir si ce(s) direc- 
teur doit être ou non pris parmi les mandataires administrateurs. Le Code de commerce de 1807 n'en disait rien, sans pour autant l'exclure, puisqu'il prévoyait que ceux-ci puissent être salariés. La loi de 1867 l'a envisagé dans une formule très confuse qui laisse le choix ouvert : les «mandataires peuvent choisir parmi eux un directeur, ou, si les statuts le permettent, se substituer un mandataire étranger dont ils sont responsables envers elle» (art. 22, al. 2). En pratique, les deux situations se retrouvent sans qu'une solution ne s'impose de manière évidente. La formule n'est généralement pas fixée par les statuts, ce qui permet au conseil d'administration d'opérer des aménagements réguliers. Première hypothèse, le directeur est choisi parmi les administrateurs ; il est alors dénommé «administrateur-directeur» ou, plus fréquemment, «administrateur délégué », titre dominant dans la pratique sans jamais avoir été officialisé. Bien que la loi ne le prévoit pas explicitement, cet «administrateur délégué » n'est pas toujours unique ; il peut y en avoir deux voire trois au sein du conseil. Quel qu'en soit le nombre, une différence apparaît clairement avec les autres administrateurs : cette fonction est exercée de manière exclusive dans une même entreprise ou, du moins, dans un même groupe ; un éventuel cumul ne se retrouve qu'entre une société mère et des sociétés filiales ou assimilées. La nomination d'un administrateur délégué par le conseil débouche généralement sur l'attribution de prérogatives de direction sur un certain nombre de services centraux. Lorsqu'il y en a plusieurs, on peut avoir un partage entre, par exemple, la direction des usines et celle de la direction des services administratifs ou commerciaux. Les grandes décisions restent $\mathrm{du}$ ressort $\mathrm{du}$ conseil, mais les administrateurs délégués ont généralement délégation pour effectuer des actes de gestion courante ${ }^{23}$, comme des achats dans la limite d'un certain montant ou des recrutements de personnel jusqu'à un certain niveau. Ce ou ces administrateurs délégués, s'ils ne sont pas nécessairement des salariés stricto sensu, reçoivent une rémunération au moins en partie fixe qui constitue généralement leur revenu principal. Il peut arriver, même si ce n'est pas l'hypothèse la plus fréquente, que ce soit le président du conseil qui soit également désigné administrateur délégué. Dans tous les cas, on a une confusion, autour de ce concept ambigu d' « administration », entre surveillance et gestion au sein d'un même organe, à la différence de la solution dualiste imposée par la législation allemande sur les sociétés par actions avec un directoire et un conseil de surveillance distincts. En France, au sein d'un même conseil, on peut avoir un ou plusieurs membres qui soient des dirigeants gérant l'entreprise à temps plein et d'autres, dont le président ou non, qui n'exercent de fait qu'une fonction de surveillance externe. Les administrateurs délégués sont ainsi amenés à participer aux votes du conseil relatifs à la ratification des décisions qu'ils ont prises ou à l'adoption des propositions qu'ils ont faites ; la confusion est à son comble lorsque c'est celuilà même qui préside les séances qui en est à l'origine. Il paraît alors difficile aux autres administrateurs de s'opposer. Cette situation pour le moins ambiguë, régulièrement déplorée par les juristes, n'a cependant, tant elle était ancrée dans les habitudes, pas fait l'objet, jusqu'en 1940, d'une réforme législative.

Une autre alternative est celle d'un « mandataire étranger » désigné à l'extérieur du conseil, qui est en pratique, à l'inverse de ce que prévoit la loi, généralement dénommé « directeur». Lui non plus n'est pas nécessairement salarié, dans la mesure où il peut n'avoir qu'un statut de mandataire social, mais il exerce également sa fonction à plein temps et de manière exclusive. Comme pour les administrateurs délégués, il peut y en avoir plusieurs qui se partagent la direction de différents services ou branches. De maniè-

${ }^{23}$ G. Droin, Les pouvoirs du conseil d'administration de la société anonyme, thèse de doctorat en droit, Paris, Émile Larose, 1912, p. 58. 
re plus fréquente après 1918 , le conseil tend à attribuer le qualificatif plus prestigieux de « général » à ce ou ces directeurs pour les distinguer des autres simples directeurs de services, qui ne sont eux que des cadres salariés. Léon Lévy, directeur de l'entreprise Châtillon-Commentry depuis 1891, a ainsi dû attendre 1920 pour avoir droit au titre de directeur général. Au-delà du caractère symbolique, cette qualification semble correspondre à une plus grande autonomie de la direction par rapport à un conseil dont le contrôle se fait moins étroit, faute de temps pour s'y consacrer suffisamment de la part de membres. Cette solution du ou des directeurs (général-aux) distincts du conseil correspond de fait à la situation dualiste allemande ; il permet une distinction plus claire entre gestion et surveillance ${ }^{24}$. Le directeur assiste bien aux séances du conseil d'administration, comme les membres du directoire allemand assistent généralement aux séances du conseil de surveillance, mais il le fait à titre consultatif, pour présenter ses actions ou projets, sans participer aux votes.

Cette solution a priori plus satisfaisante n'a jamais été encouragée par le législateur. En dehors des grandes compagnies de chemins de fer qui sont restées fidèles au système qui leur avait été imposé par le Conseil d'État lors de leur création ${ }^{25}$ jusqu'à leur nationalisation en 1937, elle n'a guère été adoptée durablement par d'autres entreprises. Les pratiques sont en fait plus confuses et irrégulières. Une entreprise comme Marine-Homécourt a eu, à partir de 1911, un directeur général, Théodore Laurent, avant de décider en 1917 de le désigner également comme administrateur ; il devient ensuite vice-président-directeur général, puis, en 1927, à la mort du titulaire d'une simple présidence, président délégué, avec un directeur général à ses côtés, toutefois. À cette confusion diachronique entre les deux formules s'ajoute une confusion synchronique possible; il est fréquent d'avoir un ou plusieurs administrateurs délégués associés à un ou plusieurs directeurs généraux. À la suite de l'absorption d'une autre entreprise, Denain-Anzin a ainsi en 1920 deux administrateurs délégués et un directeur général, un deuxième étant même nommé en 1922. En 1925, les deux administrateurs délégués deviennent vice-présidents sans délégation, mais il faut attendre 1934 pour n'avoir plus, avec le décès du premier, qu'un seul directeur général, promu depuis l'année précédente également administrateur... On a donc en fait une multiplicité de configurations, sans que leur simple description formelle permette d'en dégager des conclusions définitives en matière de répartition du pouvoir ; tout dépend des situations pratiques et des rapports de force personnels. À côté du simple administrateur qui peut apparaître, lorsqu'il n'a pas de fonction principale dans une autre entreprise, comme un simple «notable » ou « amateur» des affaires ou, lorsqu'il en a une, comme un cumulard plus ou moins lointain, on a bien émergence de la figure du dirigeant professionnel : il consacre l'essentiel de son temps à la gestion d'une seule entreprise et en tire l'essentiel de ses revenus, tout en se distinguant par son statut de mandataire du simple cadre salarié. Cette émergence se fait toutefois dans une certaine confusion à la fois sémantique et pratique : le législateur a longtemps considéré que l'organisation de la gestion de la SA relevait de la volonté souveraine des contractants ${ }^{26}$.

\footnotetext{
${ }^{24}$ Voir, parmi de multiples admirateurs des solutions allemandes, pas seulement sous l'Occupation, P. Gontier, Le directeur..., op. cit., p. 274 ou L. Czulowski, La notion de direction dans les sociétés anonymes et la législation de 1940, Toulouse, Imprimerie du Sud, 1943, p. 93 qui soulignent la « supériorité » du droit allemand pour l'organisation de la direction.

${ }^{25}$ A. Lefebvre-Teillard, La société anonyme..., op. cit., p. 266.

${ }^{26}$ A. Paviot, La réforme des sociétés anonymes. La loi du 16 novembre 1940 et ses antécédents, Lyon, Bosc Frères, M. \& L. Riou, 1941, p. 51.
} 


\section{L'absence pratique de révocation des dirigeants}

Les présidents ou les administrateurs délégués sont, en principe, comme les autres administrateurs, révocables sans indemnité par l'assemblée générale. Les directeurs (généraux) non-administrateurs sont également, en tant que «mandataires étrangers » au conseil, considérés comme liés à la société par un contrat de mandat temporaire, qui peut être dénoncé librement par les déléga$\operatorname{taires}^{27}$. Pour les distinguer des directeurs salariés, « directeurs techniques », les tribunaux s'attachent moins au titre formel qu'aux attributions effectivement conférées à l'intéressé, qui doivent correspondre à l'ensemble de celles attribuées aux administrateurs, et ne pas se limiter à une responsabilité technique précise $^{28}$. Le directeur technique est en effet mieux protégé dans la mesure où son contrat de «louage de services » ne peut être rompu sans préavis ni motif ; une rupture abusive peut donner lieu à indemnité29. Mais rien n'interdit à un directeur de cumuler les deux statuts, une pratique semble-t-il fréquente ${ }^{30}$ pour donner au mandataire une protection dont il en principe exclu. Les évictions prématurées sont toutefois rares et elles débouchent encore plus rarement sur un contentieux judiciaire. Elles sont généralement présentées comme des simples « retraites » plus ou moins anticipées. Comme pour les simples administrateurs, il n'existe en effet pas de limite d'âge légale et même conventionnelle. Les présidents sans délégation, qui n'exercent leur fonction qu'à titre accessoire, meurent souvent en scène, à des âges parfois canoniques, à l'image de Léon Molinos (Marine-Homécourt) ou de Henry Darcy (Châtillon-Commentry) chacun à 86 ans, res- pectivement en 1914 et 1926. Le même phénomène se retrouve, à un degré moindre, pour des administrateurs délégués ou directeurs généraux. Georges Vésier, président délégué de la Compagnie française des métaux, est resté en poste jusqu'à son décès en 1938 à l'âge de 80 ans. Léon Lévy a bien quitté à 73 ans en 1924 la direction générale de Châtillon-Commentry pour en devenir simple administrateur, mais il est décédé dès l'année suivante. Dans certaines entreprises, la pratique s'instaure cependant d'un abandon des fonctions opérationnelles autour de 60-65 ans, pour se contenter de présidences ou vice-présidences sans délégation. Ainsi, Léopold Pralon (Denain-Anzin) abandonne en 1930, à 75 ans, sa délégation pour n'être plus que vice-président, avant toutefois de prendre la présidence du conseil en 1935 jusqu'à sa mort en 1938. Son homologue François Villain (Nord-Est) a quitté son poste d'administrateur délégué dès l'âge de 61 ans en 1924 pour devenir, jusqu'à son décès en 1940, vice-président du conseil. Difficile de savoir si un tel retrait anticipé comportait une dimension contrainte, aucune raison de santé n'étant là invoquée ; quoi qu'il en soit, il ne correspond pas à une rupture avec la société susceptible de déboucher sur un contentieux. Même les départs forcés par la crise des années 1930 s'effectuent avec ménagements. Celui de René Robard à 67 ans en 1931 de la présidence déléguée des Tréfileries et laminoirs du Havre en difficultés financières est présenté officiellement comme volontaire : il « a demandé à ses collègues de le décharger [de la présidence] après 10 ans d'exerci$\mathrm{ce}^{31} »$; il reste membre du conseil dont il démissionne cependant l'année suivante. Ce qui apparaît aux contemporains ${ }^{32}$ comme le $^{2}$ «débarquement» de la direction des

\footnotetext{
${ }^{27}$ P. Maureil-Deschamps, De la situation respective..., op . cit., p. 63.

${ }^{28}$ Ibid., p. 70-73 ; P. Gontier, Le directeur..., op. cit., p. 31 ; L. Czulowski, La notion..., op. cit., p. 35 sq.

${ }^{29}$ P. Gontier, Le directeur..., op. cit., p. 23-25.

${ }^{30} \mathrm{~J}$. Lefebvre, Le président-directeur général. Loi du 16 novembre 1940, Paris, F. Loviton, 1941, p. 12.

${ }^{31}$ ACL, DEEF 41353, RA exercice 1930-1931, AG du 23 décembre 1931.

${ }^{32}$ Voir A. Isaac, Journal..., op. cit., 28 novembre 1932, p. 565.
} 
Aciéries de Longwy d'Alexandre Dreux en novembre 1932 est encore moins brutal : à 80 ans, celui qui avait dirigé l'entreprise de manière très personnelle pendant quarantecinq ans doit se contenter d'un titre d'administrateur président d'honneur qu'il conserve jusqu'à sa mort en 1939. Quelques rares départs plus prématurés correspondent à des réorganisations internes qui débouchent aussi sur des arrangements à l'amiable. Paulin Grandel quitte dès l'âge de 51 ans en 1919 la direction générale de Kuhlmann, à la suite du développement considérable pendant la guerre de cette entreprise chimique d'importance jusqu'alors régionale qu'il n'a pu accompagner, étant resté au siège lillois occupé par les Allemands, mais il devient administrateur et secrétaire du conseil. Henry Mayoussier est bien victime à 54 ans de la suppression en 1935 de la « direction générale commerciale des produits chimiques de Saint-Gobain », regroupée avec celle des « usines de produits chimiques » mais, malgré son titre, il n'avait qu'un statut de directeur technique ; il est, "sur sa demande», « admis à la retraite », avec une allocation de $50000 \mathrm{~F}$ par $\mathrm{an}^{33}$; il est nommé « directeur général honoraire et conseil de la Compagnie » et reçoit « à ce dernier titre, et pendant toute la durée du temps où il exercera les fonctions, une rémunération annuelle de $150000 \mathrm{~F}$ en sus de sa retraite» qui semble largement compenser la perte des revenus antérieurs ${ }^{34}$ : la nature de ces fonctions n'a jamais été précisée par le conseil. Les intéressés, surtout lorsqu'ils ne sont que mandataires, n'auraient de toute façon pas intérêt à aller au contentieux. En vertu du principe de la révocation ad nutum, les tribunaux seraient susceptibles de leur refuser une indemnisation qu'ils peuvent en revanche obtenir, en dehors de tout cadre légal, par des accords internes. Les sociétés préfèrent payer pour étouffer des conflits ouverts qui sont toujours mal perçus par les milieux financiers. Les intéressés ont besoin d'un arrangement pour maintenir leur train de vie : jusqu'à une période récente en France, il n'existait pas de véritable marché des dirigeants de haut niveau ; ils ne pouvaient donc guère espérer retrouver des fonctions semblables dans une autre entreprise.

\section{UNE RÉGLEMENTATION LÉGALE À PARTIR DE 1940 RELATIVEMENT IMPUISSANTE À CODIFIER LES PRATIQUES}

L'intervention autoritaire du législateur en 1940 n'a pas débouché sur l'adoption d'une formule unique de direction des SA ; elle a même renforcé la confusion entre direction et contrôle autour de la personne du PDG. Des réformes législatives ultérieures se sont contentées d'offrir des options supplémentaires débouchant sur une grande liberté pour les associés.

\section{La fausse rationalisation de la loi de 1940 autour du président}

Il a fallu, après que des propositions soient restées bloquées dans les navettes entre l'Assemblée nationale et le Sénat dans les années 1930, la suspension du Parlement sous Vichy pour que la loi de 1867 soit réformée par un acte dit «loi du 16 novembre $1940 »$. Ce texte promulgué rapidement ${ }^{35}$, sans travaux préparatoires ni exposé des motifs qui permettraient d'éclairer les inten-

\footnotetext{
${ }^{33}$ ASG, 2 B 28, PV du CA, séance du 26 juin 1935.

${ }^{34} \mathrm{Il}$ avait un salaire annuel minimal garanti fixé à $40000 \mathrm{~F}$, complété par un calcul complexe de pourcentages régressifs sur les résultats ; ASG, 2 B 26, PV du CA, séance du 11 janvier 1928.

${ }^{35}$ Un premier texte promulgué le 18 septembre 1940 est remplacé, avec des additions mineures, par un second le 16 novembre.
} 
tions du «législateur $»^{36}$, a, pour la première fois, cherché à rationaliser la confusion des pratiques antérieures. C'est la consécration d'une conception «institutionnelle» de la SA «selon laquelle la gestion peut et doit être organisée par la loi », «la volonté des actionnaires [ayant] cessé depuis longtemps de constituer une garantie de bonne gestion, puisque leur assemblée se trouve, en fait, sous la domination des administrateurs » qui ne servent pas nécessairement les intérêts des premiers $^{37}$. Mais cette rationalisation s'est effectuée d'une manière peu cohérente. Ce texte consacre d'abord, avec plus d'un siècle de retard sur la pratique, l'existence d'un conseil d'administration et écarte, avec une exigence minimale de trois membres, l'hypothèse marginale de l'administrateur unique. Après l'échec de plusieurs tentatives antérieures, une limitation du cumul à huit mandats d'administrateur est introduite ; une restriction supplémentaire à deux mandats, supprimée en 1953, est même prévue pour les personnes de plus de 70 ans. La grande innovation est la concentration de principe des pouvoirs de direction générale aux mains du seul président dont l'existence est aussi pour la première fois légalement reconnue ${ }^{38}$. La solution, minoritaire jusqu'alors, du président délégué, est ainsi appelée à devenir la règle, sous réserve qu'une alternative est prévue avec la possibilité, «à défaut», pour le président de déléguer «sous sa responsabilité personnelle » la direction générale à un tiers qui ne peut être membre du conseil d'administration ${ }^{39}$. Il ne s'agit pas, comme on le dit souvent, de l'adoption d'une solution allemande ${ }^{40}$, puisque la confusion direction / surveillance est justement consacrée en la personne du PDG. En revanche, cette confusion est limitée à la personne du PDG dans la mesure où « aucun autre membre du conseil ne peut être investi de fonctions de direction dans la société » (art. 2, al. 2). Les sociétés existantes se trouvent donc confrontées à différentes alternatives. Soit le président en place, délégué ou non, prend en main également la direction générale, soit il la délègue à l'un des administrateurs délégués ou directeurs généraux ; celui-ci doit alors se retirer du conseil et les éventuels autres titulaires abandonner toute fonction de direction ou renoncer à siéger au conseil pour n'être plus que directeurs techniques salariés ; soit le président renonce à son poste et c'est l'un des administrateurs délégués ou directeurs généraux qui devient PDG. Les deux situations se trouvent dans la pratique, selon les profils des uns et des autres, sous réserve que l'interdiction adoptée du cumul de plus de deux présidences en libère un certain nombre pour la seconde solution. La portée de la réforme ne doit cependant pas être surestimée ; beaucoup de présidents sans délégation restent en place en continuant de déléguer la direction générale ; cette délégation ne peut simplement plus être partagée. Des solutions intermédiaires peuvent aussi être adoptées : chez Rhône-Poulenc, le président François AlbertBuisson délègue ainsi ses pouvoirs au directeur général Nicolas Grillet, jusqu'alors coadministrateur délégué, « étant entendu qu'il se réserve la direction des services administratifs et financiers $»^{41}$. La loi reste obscure

\footnotetext{
${ }^{36}$ J. Lefebvre, Le président-directeur général..., op. cit., p. 6-7 ; A. Paviot, La réforme des sociétés anonymes..., op. cit., p. 88.

${ }^{37}$ Ibid., p. 51.

${ }^{38} \mathrm{P}$. Barbry, Le régime actuel de l'administration des sociétés anonymes, Lille, Imprimerie Douriez-Bataille, 1943 , p. 50.

${ }^{39}$ Le législateur aurait, selon un commentateur de l'époque, voulu éviter que le président et le directeur général votent différemment; ibid., p. 180.

${ }^{40} \mathrm{Ou}$ alors, comme le note un commentateur un peu zélé de l'époque, une application « encore plus rigoureuse qu'en Allemagne » du Führerprinzip, qui n'avait pas véritablement remis en cause le caractère traditionnellement collégial du directoire : L. Czulowski, La notion..., op. cit., p. 133.
}

${ }^{41}$ ADR, 146 J 9, PV du CA de Rhône-Poulenc, 27 décembre 1940. 
sur le fait de savoir qui, du président ou du conseil, doit désigner ce directeur général ; les exégètes adoptent des positions divergentes $^{42}$, d'une portée pratique limitée cependant : on imagine mal que le président fasse un choix qui irait à l'encontre de la position du conseil.

\section{La consécration effective du « PDG " par la loi de 1943}

C'est la nouvelle « loi » du 4 septembre 1943, sans employer explicitement le terme, qui consacre véritablement le «présidentdirecteur général »; l'adjonction éventuelle d'un directeur général ne décharge plus le président d'une direction générale qui lui revient de droit : «sur sa proposition, le conseil peut, pour l'assister, lui adjoindre, à titre de directeur général, soit un de ses membres, soit un mandataire choisi hors de son sein » (art. 13. al. 2). Le texte tranche également le débat de savoir qui nomme cet adjoint en confiant plus explicitement cette prérogative au conseil sur la proposition du président. Une autre innovation, appréciée par les intéressés, est la possibilité que le directeur général puisse, à nouveau, être également administrateur, ce qui revient de fait à rétablir l'ancien administrateur délégué, avec la seule contrainte qu'il soit unique ${ }^{43}$. D'une multiplicité de configurations auparavant, on arrive donc à une simple alternative entre concentrations des pouvoirs au profit du seul PDG ou au profit d'un duo PDG / directeur général (administrateur ou non) ; les autres directeurs ne sont que des directeurs techniques salariés exclus du conseil, en principe dotés, au mieux, d'un titre de «directeur général adjoint ». Dans la pratique, la deuxième option, présentée comme l'exception par le législateur, semble avoir été plutôt dominante, dans la continuité des situations antérieures ${ }^{44}$. L'éventail des possibles est restreint, mais la confusion entre direction et surveillance consacrée, dans la mesure où les administrateurs non directeurs restent sous la présidence de celui dont ils devraient justement surveiller la gestion, sans maîtrise donc du calendrier des séances et de l'ordre du jour du conseil.

Quelle que soit la solution adoptée, unique ou duale, la tendance ultérieure est celle d'un renforcement de l'engagement du PDG dans la gestion de l'entreprise. La limitation du cumul à deux présidences a rendu certains gros cumulards plus disponibles. L'accession à la présidence s'est de plus en plus effectuée par la filière de la direction interne plutôt que par celle du seul conseil d'administration. Elle devient ainsi la consécration d'un cursus honorum marquée par des étapes successives bien balisées, directeur, directeur général adjoint, directeur général, administrateur directeur général, vice-PDG, puis PDG. De grandes entreprises en finissent à la fin des années 1950 avec ces présidents « grands notables » des affaires au profit de managers exclusifs. René Piaton qui, jusqu'à la nationalisation de 1946, gérait par ailleurs son groupe familial lyonnais de gaz et d'électricité et qui a longtemps (19321951) également présidé l'importante Compagnie électro-mécanique, laisse ainsi, à son décès en 1958 à l'âge de 74 ans, la présidence de Pechiney au vice-PDG Raoul de Vitry, en poste dans l'entreprise depuis 1929. De même, François Albert-Buisson, qui par-

\footnotetext{
${ }^{42}$ Le directeur général serait librement choisi par le président d'après A. Paviot, La réforme des sociétés anonymes..., op. cit., p. 111 ou P. Barbry, Le régime..., op. cit., p. 176 ; en revanche, J. Lefebvre, Le président-directeur général..., op. cit., p. 53, considère que, dans l'incertitude de la loi, c'est « apparemment le conseil » qui devrait le désigner.

${ }^{43}$ Une interprétation divergente selon laquelle la loi autoriserait le président à se faire assister de plusieurs directeurs généraux (voir notamment L. Czulowski, La notion..., op. cit., p. 173) reste minoritaire et n'est pas retenue par la pratique.

${ }^{44} \mathrm{G}$. Vernon, Le président-directeur général dans les sociétés anonymes, thèse de doctorat, faculté de droit de Paris, LGDJ, 1952, p. 13.
} 
tageait sa présidence de Rhône-Poulenc avec la gestion de ses propres laboratoires pharmaceutiques et l'écriture d'essais qui lui ont valu d'être élu à l'Académie française en 1955, se retire en 1959, à 78 ans, au profit du vice-PDG Marcel Bô, qui fait carrière dans l'entreprise depuis 1920. L'adoption progressive, avant que le législateur ne tente à nouveau de s'en emparer timidement dans les années $1970^{45}$, d'une limite d'âge conventionnelle qui limite les responsabilités susceptibles d'être exercées par des septuagénaires ou des octogénaires, favorise aussi la présence de présidents plus actifs. Les années 1960 et suivantes sont marquées par la prédominance du PDG tout puissant, que la nouvelle législation sur les sociétés anonymes de la loi de 1966 n'a pas remise en cause.

\section{La réforme timide de 1966 ne fait qu'ouvrir le champ des possibles}

Le projet de loi initial se contente de prévoir que, dans les sociétés importantes, un deuxième directeur général peut être désigné. Mais le rapporteur du texte à l'Assemblée nationale, l'avocat et député François Le Douarec (Ille-et-Vilaine), s'est appuyé sur les critiques récurrentes de la confusion entre direction et contrôle qui tendent à souligner la supériorité du droit allemand $^{46}$ pour proposer, dans un amendement présenté en commun avec le président de la Commission des lois René Capitant, l'introduction du système du directoire et du conseil de surveillance ${ }^{47}$. Mais il n'a pas osé imposer cette solution : " Il n'est pas possible d'introduire brusquement dans notre pays, de façon impérative, et sans transition, des institutions étrangères qui, bien qu'assurément meilleures, risqueraient de heurter nos habitudes, sinon nos traditions. » Face à l'hostilité ouverte du CNPF en particulier, la disposition n'est proposée et adoptée par le Parlement que sous la forme d'une option alternative offerte aux parties. Elle n'a d'ailleurs connu pendant longtemps qu'un succès limité dans les grandes entreprises. Peugeot SA est, à partir de 1972, la seule à y avoir recours durablement, parce qu'elle lui permet une séparation entre un directoire confié plutôt à des managers et un conseil de surveillance contrôlé par la famille actionnaire. La pratique moniste du PDG associé à un ou deux directeurs généraux souvent administrateurs eux-mêmes reste largement dominante. La seule limite à la confusion entre direction et contrôle est l'obligation introduite par le législateur de 1966 que « le nombre des administrateurs liés à la société par un contrat de travail ne peut dépasser le tiers des administrateurs en fonction » (art. 93, al. 2). Les autres n'en sont pas pour autant «indépendants » au sens restrictif adopté par une convention récente des milieux patronaux (code AFEP-MEDEF), dans la mesure où ils peuvent entretenir d'autres liens avec l'entreprise (anciens salariés, salariés de filiales $)^{48}$. Le problème subsiste que le conseil, ainsi présidé et composé, n'est pas en mesure d'exercer, avec l'extériorité requise, le contrôle de la direction.

\footnotetext{
${ }^{45}$ Une loi du 31 décembre 1970 se contente de prévoir que, « à défaut de disposition expresse des statuts, le nombre des administrateurs ayant dépassé l'âge de 70 ans ne pourra être supérieur au tiers des administrateurs en fonction »... ${ }^{46}$ Il fait notamment référence au livre d'un «maître du droit commercial », G. Ripert, Aspects juridiques du capitalisme moderne, Paris, LGDJ, $1^{\text {re }}$ éd. 1946.

${ }^{47}$ Rapport de François Le Douarec, Assemblée nationale, $1^{\text {èr }}$ lecture, Journal officiel de la République française (JORF), Documents de l'Assemblée nationale, $\mathrm{n}^{\circ} 1368,14$ mai 1965, p. 695-696.

${ }^{48}$ Sans compter les actionnaires significatifs également exclus par cette définition mais qui peuvent, eux, être considérés comme en mesure d'exercer un contrôle extérieur. Sur les origines de ce code, cf. F. Bancel, « Directions in French corporate governance since 1945 », Entreprises et Histoire, $n^{\circ} 21$, juin 1999, p. 53-57.
} 


\section{Le retour possible à la pratique d'avant 1940 introduit en 2001}

La seule innovation législative marquante intervenue depuis 1966 est celle de $2001^{49}$ qui prévoit, outre une restriction supplémentaire du cumul des mandats d'administrateur à cinq, la possibilité de revenir à la situation dominante avant 1940 d'un président non exécutif qui n'exerce plus la direction générale, laissée à un ou plusieurs directeurs généraux, administrateurs ou non. L'idée est, sans remettre en cause le conseil d'administration comme organe unique, de mieux distinguer en son sein ceux qui dirigent de ceux qui contrôlent en donnant la possibilité au président de n'être qu'un contrôleur. Si le projet initial tendait à privilégier cette option, le texte laisse finalement les deux solutions sur le même $\operatorname{plan}^{50}$. Cette solution connaît rapidement un certain succès, puisqu'elle est ainsi adoptée dès 2004 par huit sociétés du CAC 40 - elle permet surtout à des PDG de retarder leur retrait définitif des affaires en se repliant d'abord sur la seule présidence -, alors que l'option dualiste à l'allemande introduite en 1966 s'est entre-temps également répandue ( 7 cas). Mais, une nouvelle fois, le législateur, pour ne rien imposer, s'est contenté d'offrir une option supplémentaire. La direction des grandes entreprises se trouve donc éclatée entre trois configurations possibles, sans compter la survivance historique des SCA (2 cas dans le CAC 40).

$$
* * *
$$

La conception institutionnelle de la SA s'est bien imposée en apparence sur celle de la liberté contractuelle : en raison des enjeux économiques et sociaux dont les grandes entreprises sont porteuses, leur direction rebaptisée «gouvernance » est considérée aujourd'hui comme relevant d'un intérêt général, au-delà même de la seule protection des actionnaires ; elle est donc devenue un objet de droit, dont s'est emparé le législateur à plusieurs reprises, avec l'introduction dans des textes de plus en plus étoffés de dispositions d'ordre public, mais pour un résultat finalement maigre : on se retrouve avec une diversité des pratiques possibles à peu près aussi grande que celle qui existait avec les dispositions laconiques des textes de 1807 et 1867 ; la sophistication croissante du droit a paradoxalement réintroduit la liberté des associés. Le législateur admet ainsi implicitement son impuissance : la qualité d'une gouvernance devrait moins à l'option légale retenue qu'à son fonctionnement effectif; les meilleures solutions formelles ne fonctionnent efficacement que si les « surveillants » ont effectivement les moyens et la volonté d'exercer leur contrôle de manière indépendante, ce qu'aucun texte ne suffit à imposer.

\footnotetext{
${ }^{49}$ Loi sur les «nouvelles régulations économiques » du 15 mai 2001, JORF, 16 mai 2001, p. 7776.

${ }^{50}$ A. Viandier (avec F. Mansuy), « Administration. Direction. Président-directeur-général », Jurisclasseur, Fasc. 13310,2001, p. 4.
} 